\title{
Combined effects of long-pulsed neodymium-yttrium-aluminum-garnet laser, diprospan and 5-fluorouracil in the treatment of keloid scars
}

\author{
XIAO-E CHEN ${ }^{1,2}$, JUAN LIU $^{1}$, AFZAAL AHMED BIN JAMEEL ${ }^{1}$, MAYA VALESKA $^{1}$, \\ JIA-AN ZHANG ${ }^{1}$, YANG XU ${ }^{1}$, XING-WU LIU ${ }^{2}$, HONG ZHOU ${ }^{2}$, DAN LUO ${ }^{1}$ and BING-RONG ZHOU ${ }^{1}$ \\ ${ }^{1}$ Department of Dermatology, The First Affiliated Hospital of Nanjing Medical University, Nanjing, Jiangsu 210029; \\ ${ }^{2}$ Department of Dermatology and Venereology, Nanjing Jingdu Hospital, Nanjing, Jiangsu 210002, P.R. China
}

Received December 13, 2015; Accepted January 20, 2017

DOI: $10.3892 / \mathrm{etm} .2017 .4438$

\begin{abstract}
Keloids are benign tumors that originate from scar tissues, but they usually overgrow beyond the original wounds In a three-month single-center clinical trial, 69 patients were randomly divided into three groups. Patients in group 1 were treated with intralesional injection of diprospan $(2 \mathrm{mg}$ betamethasone disodium phosphate and $5 \mathrm{mg}$ betamethasone dipropionate in $1 \mathrm{ml}$ ) with one-month intervals for three months. Patients in groups 2 and 3 were injected with a combination of $0.5 \mathrm{ml} 5$-fluorouracil (5-FU; $25 \mathrm{mg} / \mathrm{ml})$ and diprospan as above for three months also. Prior to each injection, the keloids of patients in group 3 were additionally irradiated by a 1,064-nm neodymium-yttrium-aluminum-ga rnet (Nd:YAG) laser with a single pulse at an energy density of $90-100 \mathrm{~J} / \mathrm{cm}^{2}$ and a pulse width of $12 \mathrm{msec}$. Clinical responses were evaluated by patient self-assessment and overall assessment by an observer according to the clinical signs of erythema, pruritus and pliability. A total of sixty-two patients completed the tests of the present study. At 2 and 3 months, the patients in all treatment groups showed an acceptable improvement in nearly all measurements. At the end of the study, the erythema and toughness score was significantly reduced and itch reduction was significantly greater in the diprospan + 5-FU + Nd:YAG group when compared to those in the other groups $(\mathrm{P}<0.05$ for all indexes). The acceptable responses (good to excellent improvements) reported by blinded observers were as follows: $12 \%$ in the diprospan group, $48 \%$ in the diprospan $+5-\mathrm{FU}$ group and $69 \%$ in the diprospan + 5-FU + Nd:YAG group. All of the results indicated
\end{abstract}

Correspondence to: Professor Bing-Rong Zhou or Professor Dan Luo, Department of Dermatology, The First Affiliated Hospital of Nanjing Medical University, Nanjing, Jiangsu 210029, P.R. China

E-mail: bingrong.2002@163.com

E-mail: daniluo2013@njmu.edu.cn

Key words: diprospan, 5-fluorouracil, neodymium-yttrium-aluminum-garnet laser, keloid, treatment that the combination of diprospan $+5-\mathrm{FU}+\mathrm{Nd}$ :YAG was the most efficacious therapy for keloid scars.

\section{Introduction}

Keloids are benign tumors that originate from scar tissues, but they usually extend beyond the original wounds (1). Patients with keloids often experience severe physical (deformities, restricted range of motion, pain and pruritus) and psychological (cosmetic concern) constraints (2). Although the exact pathogenesis has remained to be completely elucidated, it is generally thought that keloids are caused by abnormal responses of connective tissue after skin trauma. Thus, the suppression of an overwhelming and uncontrolled fibroblast activity in keloid may be critical for the treatment of such abnormal wound responses (3). Keloid is also characterized by increased vascularity $(4,5)$.

Keloids have been treated using various therapies with varying efficacy (6-8). There is no universally acknowledged approach to keloid scar ablation. Intra-lesional injection of steroids is one of the gold standards for keloid therapy. Corticosteroid therapy aids in inhibiting and breaking down lesions by inhibiting fibroblast growth and accelerating collagen degradation (7). Betamethasone preparation (diprospan; Schering-Plough, Kenilworth, NJ, USA) is a novel drug formulation that has been introduced to treat various types of skin disease, including keloids (9). A 1-ml ampoule of diprospan contains $2 \mathrm{mg}$ betamethasone disodium phosphate and $5 \mathrm{mg}$ betamethasone dipropionate. It is a formulation containing short- and long-term acting components. 5-Fluorouracil (5-FU) is a pyrimidine analog with anti-metabolite activity, which inhibits fibroblast proliferation $(10,11)$. 5-FU also inhibits the expression of type-I collagen gene in human keloid fibroblasts (12). The effect of long-pulsed 1,064-nm neodymium-ytt rium-aluminum-garnet (Nd:YAG) laser is based on suppression of the neovascularization process in these pathological scars (4).

The present study compared the clinical responses of keloids to treatment by intralesional injection of diprospan alone, diprospan combined with 5-FU, or diprospan combined with 5-FU and long-pulsed 1,064-nm Nd:YAG laser, in order to identify an optimal treatment strategy for keloids. 


\section{Materials and methods}

Study design. The present study was approved by the Ethics Committee of the First Affiliated Hospital of Nanjing Medical University (Nanjing, China). All participants are informed according to the Declaration of Helsinki. The keloid was diagnosed according to the criteria described in the literature (13). A total of 69 keloid patients who presented at the First Affiliated Hospital of Nanjing Medical University (Nanjing, China) from May 2014 to May 2015 were included in the present study. Patients who were treated for keloids within the last 6 months, pregnant women or women considering pregnancies in the near future, lactating women, patients with chronic renal failure or those showing any abnormal liver function or blood cell counts were excluded from the study. Furthermore, the lesions were required to be at least $10 \mathrm{~mm}$ in length and only one lesion (preferably on the trunk or proximal extremities) per patient was treated.

The keloids were injected using a syringe needle (diameter, $0.45 \mathrm{~mm}$ ) until slight blanching was visible and the maximum volume of injection per square centimeter did not exceed $0.5 \mathrm{ml}$. The dosage was modified according to the degree of lesions but the final dosage did not exceed $2 \mathrm{ml}$ per session. The tough portion of the keloids can be injected several times, separated by approximately $1 \mathrm{~cm}$. The patients were randomly divided into three study groups: Patients in group 1 (diprospan) were treated once a month by intralesional injection of diprospan $(1 \mathrm{ml} /$ ampoule contains $2 \mathrm{mg}$ betamethasone disodium phosphate and $5 \mathrm{mg}$ betamethasone dipropionate) for three months. Patients in group 2 (diprospan + 5-FU) received a combination of $0.5 \mathrm{ml} 5-\mathrm{FU}$ ( $25 \mathrm{mg} / \mathrm{ml}$; Jinyao Pharmaceutical Group, Tianjin, China) and $1 \mathrm{ml}$ diprospan was injected monthly for three times. Patients in group 3 (diprospan $+5-\mathrm{FU}+\mathrm{Nd}$ :YAG) were treated by a 1,064-nm Nd:YAG (Lumenis One; Lumenis, Santa Clara, CA, USA) with a single 12 -msec pulse at an energy density of $90-100 \mathrm{~J} / \mathrm{cm}^{2}$ with a $6-\mathrm{mm}$ spot and a single pass of spots overlapping 5-10\% without cooling for three sessions at a one-month interval. Each session consisted of three passes unless the patient felt intolerable pain at the second pass, and under this condition, the session was ceased. The session would be terminated even if the patients did not feel any pain. Local anesthesia was not applied to any of the patients. Intralesional injection of diprospan + 5-FU was similar to that in group 2 and was given immediately after each Nd:YAG treatment for a total of three sessions.

The efficacies of treatments were evaluated according to patients' satisfaction, observations and measurements of a blinded observer (an in-house dermatologist) as well as the initial and final photographic records. During the experiment, the observer compared the images, measured the erythema, toughness and pruritus of the lesions, as well as any adverse events encountered during the treatments.

\section{Evaluation procedures}

Patient self-assessment. Representative images of keloid scars at baseline and after 3 months of treatment are shown in Fig. 1. One month after each session (at the first, second, and third months of the study), the overall improvement was subjectively evaluated by the patients on a 5-point scale (14), i.e., no improvement, poor improvement $(<25 \%)$, fair improvement (26-50\%), good improvement (51-75\%) and excellent improvement (76-100\%).

Observer assessment. One month after each session (at the first, second, and third month of the study), the overall improvement was assessed by the observer by comparing the standardized photographs taken at the 4 -week intervals. The scales of improvement were similar to those of the patient self-assessment.

Erythema. Erythema was ranked by the observer on a 5-point scale as follows: $0,1,2,3$ and 4 represents no, mild, severe and very severe erythema, respectively. The percentage of lesion improvement was defined as the percentage of erythema reduction compared to the erythema at baseline.

Toughness. Toughness was evaluated by the observer on a 5-point scale as follows: $0,1,2,3$ and 4 represents no, mild, moderate, severe and very severe induration, respectively. The percentage of lesion malacia was defined as the percentage of reduction to the baseline pliability.

Pruritus. Severity of pruritus was ranked by the patients on a 5-point scale according to the following scale: 0, 1,2, 3 and 4 represent no, mild, moderate, severe and very severe pruritus, respectively. The percentage of itch reduction was defined as the percentage of pruritus reduction to the baseline pruritus.

Statistical analysis. The data were analyzed using the SPSS 11.5 software package for Windows (SPSS Inc., Chicago, IL, USA). Standard two-tailed and paired Student's t-tests were used to compare the differences between values at time-points of assessment and at baseline. One-way analysis of variance followed by post hoc and $\chi^{2}$ tests were used to compare the difference between baseline and one, two and three months. All statistical tests were two-tailed. $\mathrm{P}<0.05$ was considered to indicate a significant difference.

\section{Results}

Baseline characteristics. Sixty-two of the 69 patients who were enrolled completed the entire 12-week study. Fifty-eight percent of the patients enrolled in the study were female. The mean age of the patients was $26.7 \pm 7.5$ years and the mean duration of the lesions was $32.9 \pm 27.6$ months. A total of $4.7 \%$ of the lesions were on the face and neck, $67.8 \%$ on the trunk, $22.5 \%$ on proximal extremities and $5 \%$ on distal extremities. No statistically significant differences were observed between the study groups regarding mean age, duration as well as erythema, pruritus and pliability at baseline (Table I).

Patient self-assessment. Improvements were rated highest in the diprospan + 5-FU + Nd:YAG group and lowest in the diprospan group (Fig. 2). At the third month of the study, good to excellent improvements (by $50 \%$ or higher) were reported by a number of patients, i.e. $20 \%$ in the diprospan group, $58 \%$ in the diprospan $+5-\mathrm{FU}$ group and $78 \%$ in the diprospan $+5-\mathrm{FU}+\mathrm{Nd}: \mathrm{YAG}$ group.

Observer assessment. At the third month of the study, observer assessment results indicated that improvements also rated highest in the diprospan + 5-FU + Nd:YAG group and lowest in the diprospan group (Fig. 3). Good to excellent 
Table I. Demographic features and baseline characteristics.

\begin{tabular}{|c|c|c|c|c|}
\hline Variable & $\begin{array}{c}\text { Group } 1 \\
\text { (diprospan) }\end{array}$ & $\begin{array}{c}\text { Group } 2 \\
\text { (diprospan+5-FU) }\end{array}$ & $\begin{array}{c}\text { Group } 3 \text { (diprospan+ } \\
\text { 5-FU+Nd:YAG) }\end{array}$ & P-value \\
\hline Age (years) & $27.2 \pm 6.4$ & $26.5 \pm 7.5$ & $26.5 \pm 9.5$ & 0.872 \\
\hline Duration (months) & $29.8 \pm 25.6$ & $29.2 \pm 33.6$ & $39.8 \pm 26.8$ & 0.557 \\
\hline Erythema score & $3.5 \pm 0.8$ & $3.7 \pm 0.7$ & $3.6 \pm 0.8$ & 0.401 \\
\hline Pruritus score & $3.4 \pm 0.6$ & $3.3 \pm 0.5$ & $3.5 \pm 0.7$ & 0.628 \\
\hline Pliability score & $3.0 \pm 1.1$ & $2.9 \pm 1.0$ & $2.9 \pm 0.9$ & 0.664 \\
\hline
\end{tabular}

Values are expressed as the mean \pm standard deviation. No statistically significant differences were identified between groups (P>0.05). 5-FU, 5-fluorouracil; Nd:YAG, neodymium-yttrium-aluminum-garnet laser.

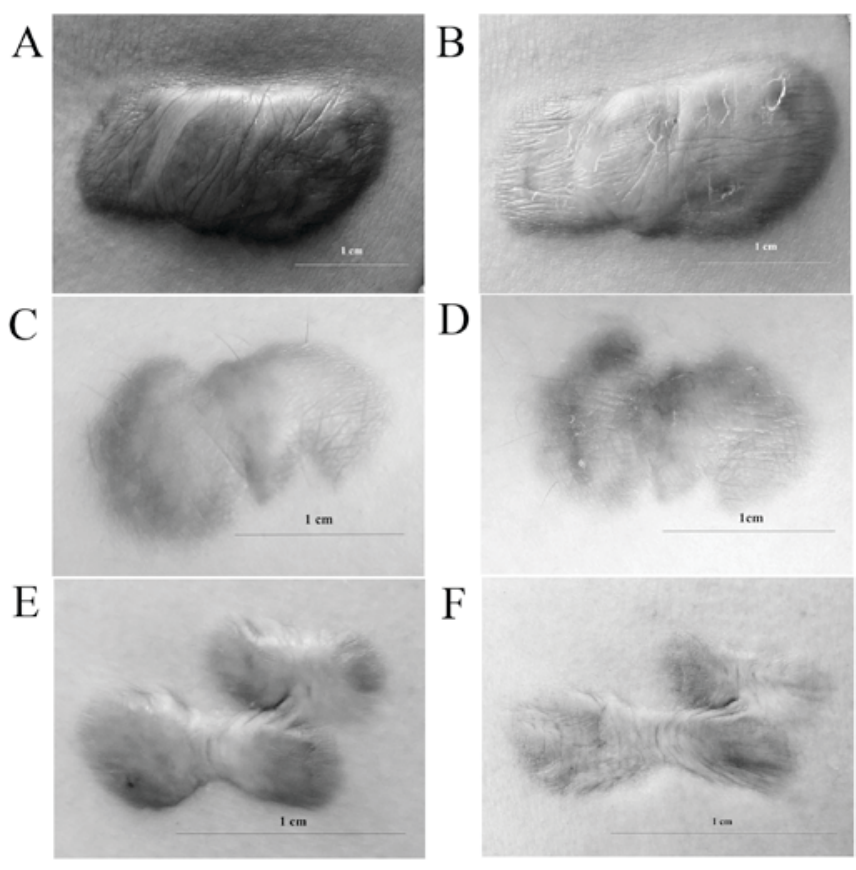

Figure 1. Representative images of keloid scars at baseline and after three months of treatment. Keloid scar from a patient from the diprospan group (A) at baseline and (B) after treatment, a patient from the diprospan + 5-FU group (C) at baseline and (D) after treatment, and a patient from the diprospan + 5-FU + neodymium-yttrium-aluminum-garnet laser group (E) at baseline and (F) after treatment. 5-FU, 5-fluorouracil.

responses were observed in $12 \%$ of the diprospan group, $48 \%$ of the diprospan $+5-\mathrm{FU}$ group and $69 \%$ of the diprospan + 5-FU + Nd:YAG group.

Erythema. The reduction in erythema score was statistically significant among all of the study groups during the entire study period $(\mathrm{P}<0.05)$. At the second month, the erythema score in the diprospan group was significantly higher than that in the diprospan +5 -FU group and the diprospan $+5-\mathrm{FU}+\mathrm{Nd}$ :YAG $(\mathrm{P}<0.05$ for each). At the third month of the study, the erythema score in the diprospan + 5-FU + Nd:YAG group was significantly lower than that in the diprospan and diprospan +5 -FU groups $(\mathrm{P}<0.05$ for each) (Fig. 4). Erythema score reduction percentages at the second and third month in the diprospan +5 -FU and the

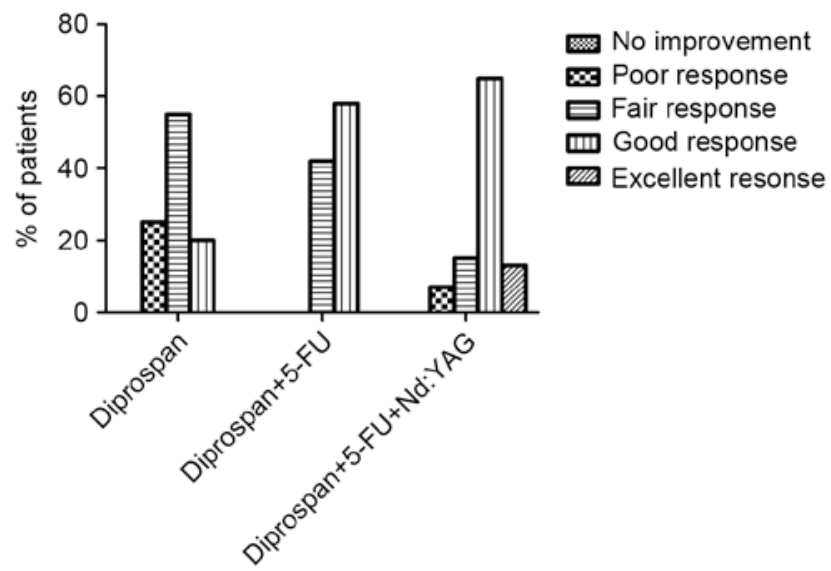

Figure 2. Response of keloid scars to the treatments at 3 months, as determined by patient self-assessment. 5-FU, 5-fluorouracil; Nd:YAG, neodymiumyttrium-aluminum-garnet laser.

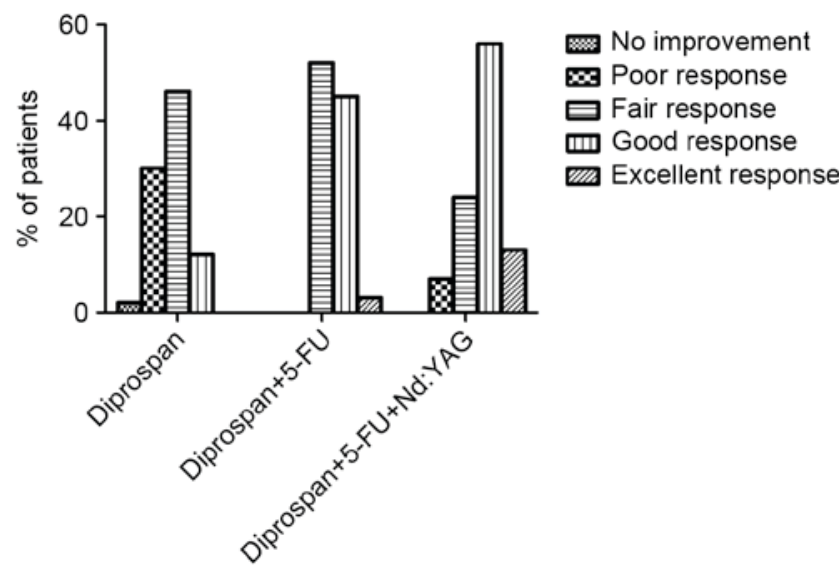

Figure 3. Response of keloid scars to the treatments at 3 months, as determined by a blinded observer. 5-FU, 5-fluorouracil; Nd:YAG, neodymiumyttrium-aluminum-garnet laser.

diprospan + 5-FU + Nd:YAG groups were significantly higher than those in the diprospan group.

Toughness. A statistically significant malacia of lesions was observed in all three study groups at the second and third month when compared to the toughness at baseline $(\mathrm{P}<0.05$ for 


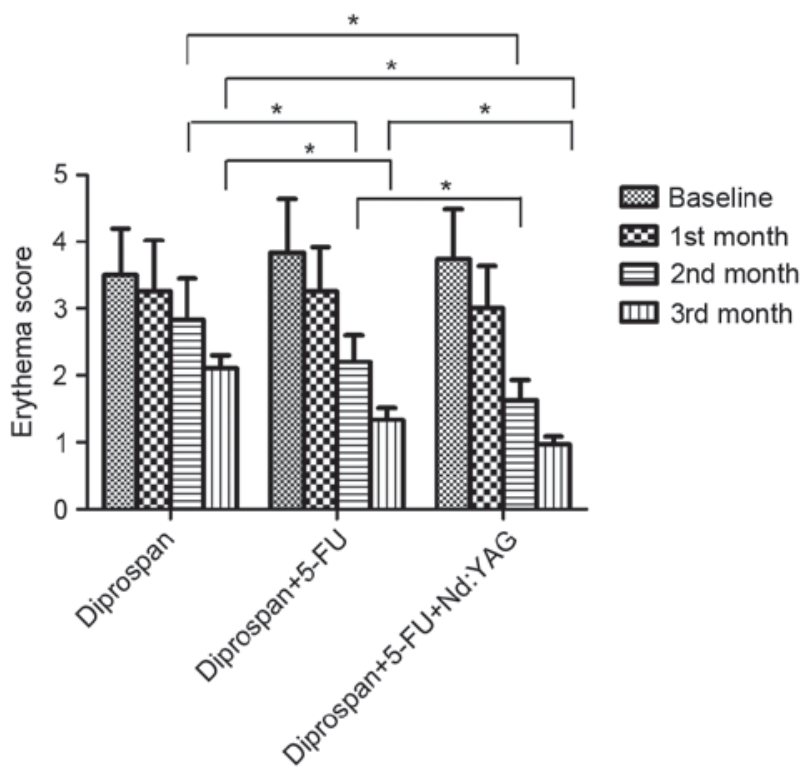

Figure 4. Effect of the keloid scar treatments on erythema in the three groups. "P<0.05. 5-FU, 5-fluorouracil; Nd:YAG, neodymium-yttrium-aluminumgarnet laser.

all) (Fig. 5). A progressive increase in lesion malacia was also observed. At the third month of the study, the pliability score in the diprospan $+5-\mathrm{FU}+\mathrm{Nd}$ :YAG group was significantly lower than that in the diprospan and diprospan +5 -FU groups $(\mathrm{P}<0.05$ for each) (Fig. 5).

Pruritus. The severity of pruritus was significantly reduced among all the study groups at all time-points when compared to that at baseline ( $\mathrm{P}<0.05$ for all) (Fig. 6). At the second and third month, there was a statistically significant reduction in pruritus score in the diprospan + 5-FU + Nd:YAG group when compared with that in the diprospan group $(\mathrm{P}<0.05)$. At the same visit, the percentage of itch reduction in the diprospan $+5-\mathrm{FU}+\mathrm{Nd}: \mathrm{YAG}$ group was significantly higher than that in the diprospan group $(\mathrm{P}<0.05)$.

Adverse events. Almost all injections were painful. In the diprospan group, $36 \%$ of patients experienced a certain degree of skin atrophy and telangiectasia. In the diprospan + 5-FU + Nd:YAG group, the site treated by Nd:YAG became purpuric, which lasted for 7-10 days. Neither adverse textural nor pigmentary alterations were observed in the diprospan +5 -FU or the diprospan + 5-FU + Nd:YAG group. Ulcers or erosions were also not observed.

\section{Discussion}

The efficiency of corticosteroid injection for keloid treatment has been well documented. Over the years, various potent glucocorticoids such as dexamethasone (15), triamcinolone (16) and betamethasone (17) have been used for intralesional injection as a treatment for keloids. An ideal preparation should rapidly take effect, while also having long-lasting effects. Therefore, a combination of long- and short-acting glucocorticoids is able to achieve improved effects. It has been reported that injection of a mixture of dexamethasone (short-acting) and

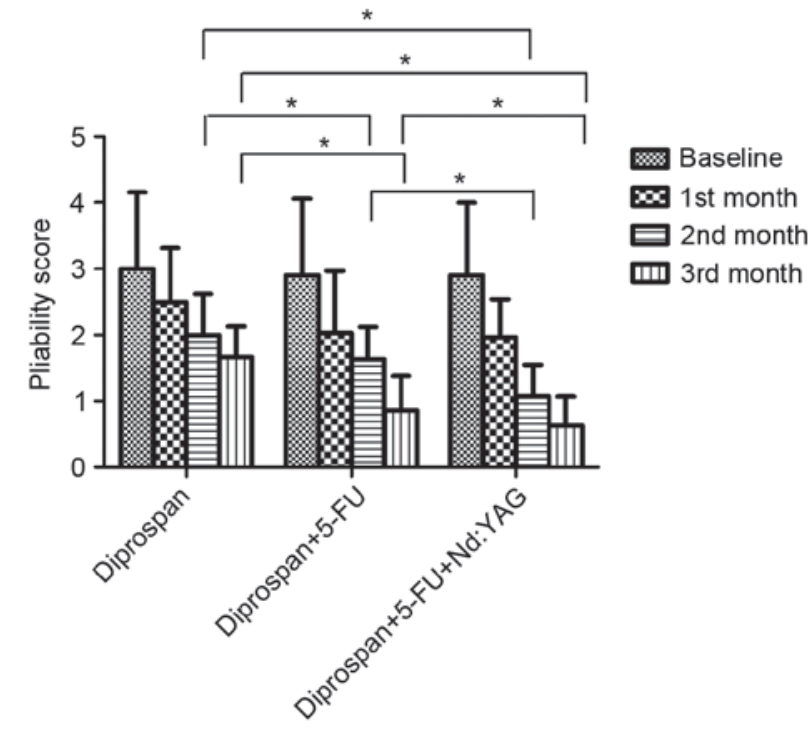

Figure 5. Effect of the treatments on keloid scar toughness in the three groups ${ }^{*} \mathrm{P}<0.05$. 5-FU, 5-fluorouracil; Nd:YAG, neodymium-yttrium-aluminumgarnet laser.

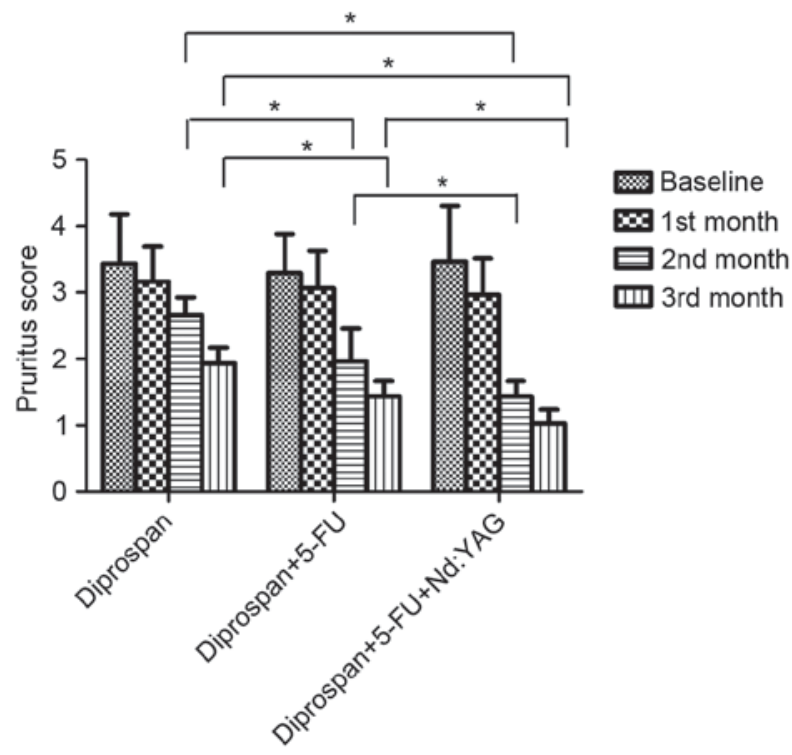

Figure 6. Effect of the treatments on pliability of treated keloid scars in the three groups. " $\mathrm{P}<0.05$. 5-FU, 5-fluorouracil; Nd:YAG, neodymium-yttriumaluminum-garnet laser.

triamcinolone (long-acting) is able to achieve ideal results in periorbital hemangioma (18). Diprospan used in the present study is a formulation containing the short-acting betamethasone disodium phosphate and the long-acting betamethasone dipropionate. Soluble betamethasone disodium phosphate acts shortly after injection, whereas betamethasone dipropionate is stored in the body and is absorbed slowly, thus providing a long-lasting (3-4 weeks) effect. Furthermore, diprospan is a convenient compound instant for use.

In the present study, treatment with intralesional diprospan alone achieved a statistically significant decrease in keloid-associated pruritus and erythema, and improved pliability, compared with baseline. In addition, subjective and objective improvements in keloid appearance were also noted 
in patients treated with intralesional diprospan. In line with a previous study, the present study further confirmed that the intralesional corticosteroid injection is an effective method to treat keloid scars (19). Numerous mechanisms by which corticosteroids influence scar formation have been reported, including reduction of fibroblast proliferation, suppression of components involved in the inflammatory response, attenuation of pro-collagen and ground substance synthesis, and decreased endothelial budding (6). Despite the benefits of intralesional corticosteroids, several adverse effects have been reported, including altered pigmentation, telangiectasia, skin atrophy, injection pain, ulceration and cushingoid habitus (20). It has been suggested that the combined use of intralesional 5-FU and low-dose corticosteroid may lead to fewer intolerable side effects when compared to intralesional corticosteroid monotherapy (10).

When compared with the diprospan group, the diprospan +5 -FU combination was more effective and provided a more rapid response. The results of the present study are consistent with those of previous ones, one of which reported that the effectiveness rate of 5-FU injection alone was $62.5 \%$, whereas the effectiveness of combined 5-FU and glucocorticoids was significantly higher at $92 \%(21,22)$. Intralesional 5 -FU is generally well tolerated. The frequently encountered adverse effects include pain at the injection site, ulceration, burning and hyperpigmentation (23-25). Systemic injection of 5-FU can cause anemia, leukopenia and thrombocytopenia. However, intralesional injection of up to $25 \mathrm{mg}$ of 5-FU or even a high dosage do not lead to these systemic complications of 5-FU (10,23).

In 1984, Abergel et al (26) reported the first clinical trial and an in vitro study that showed a reduction of collagen production after Nd:YAG laser treatment. A clinical study from 1988 by Sherman and Roselfeld (27) also reported that $\mathrm{Nd}$ :YAG laser decreases redness and induration. Although the 585-nm pulsed-dye laser (PDL) has been widely employed to treat scars by harnessing the effect of coagulation, the significance of long-pulsed Nd:YAG laser in keliod treatment has been recognized. The limited penetration depth of the yellow light emitted by PDL may also induce a resistance to further PDL treatment due to optical absorption and scattering in the epidermis and dermis at a depth of 1-2 mm (28). Thus, deeper vessels can be selectively treated by the 1,064-nm Nd:YAG laser (29,30). Indeed, due to its deeper penetration and lower absorption by hemoglobin, the 1,064-nm Nd:YAG laser has proven to be a more useful light source for the treatment of deeper or thicker vascular lesions than the traditionally used short-wavelength light sources. In a recent study, Koike et al (4) determined the efficiency of contact-mode 1,064-nm Nd:YAG laser for treating keloids and hypertrophic scars. They did not find any deterioration in the hypertrophic scars or keloids, and three of the 34 anterior chest keloids $(8.8 \%)$ did not show any response. Six months after the cessation of laser treatment, 1 of the abdominal hypertrophic scars (4\%), 18 of the anterior chest keloids (52.9\%), 5 of the upper arm keloids (35.7\%) and 4 of the scapula keloids $(25 \%)$ occurred again. Therefore, the efficacy of long-pulsed Nd:YAG laser should be improved when it is used to treat keloids. The results of the present study revealed that the combined diprospan + 5-FU + Nd:YAG treatment protocol showed higher efficacy over diprospan and diprospan +5 -FU treatment. The results suggested that the combined application of 1,064-nm $\mathrm{Nd}$ :YAG laser and drug injection for keloid treatment can achieve better clinical outcomes than conventional ones. Another main benefit derived from the pre-treatment with Nd:YAG laser was the product of an immediate oedematous reaction, which facilitated the placement of the intralesional drug injection, and consequently minimized extralesional osmosis and subsequent atrophy. It also reduced the pain associated with drug injection, probably due to the lower amount of pressure required for injection.

A main shortcoming of the present study was the short follow-up period, i.e. only three months. As Manuskiatti and Fitzpatrick reported that continuous improvement was maintained for 10-12 weeks in their study (31). It is likely that multiple successive laser treatments and a long follow-up time may be essential for achieving and identifying a better clinical response. In the present study, the diprospan + 5-FU + Nd:YAG combination was more effective than diprospan and diprospan $+5-\mathrm{FU}$, and the laser group also showed a more rapid response with less side effects. These findings thus suggested that laser treatment and combined drug injection may have a synergistic effect on keloid scars.

In conclusion, to the best of our knowledge, the present study was the first randomized clinical study to compare the effects of combined diprospan $+5-\mathrm{FU}+\mathrm{Nd}$ :YAG with diprospan alone or with diprospan $+5-\mathrm{FU}$ in the treatment of keloid scars. Subjective as well as objective assessments showed marked improvements in all measures. The effects in the diprospan $+5-\mathrm{FU}+\mathrm{Nd}$ :YAG group were more significant than those of any other treatment. The preferred approach was the diprospan + 5-FU + Nd:YAG combination, as it was proven to be the best therapy for the treatment of keloid scars.

\section{Acknowledgements}

This work was supported by grants from the National Natural Science Foundation of China (nos. 81573072 and 81371757) and the Priority Academic Program Development of Jiangsu Higher Education Institutions (no. JX10231801).

\section{References}

1. Shaffer JJ, Taylor SC and Cook-Bolden F: Keloidal scars: A review with a critical look at therapeutic options. J Am Acad Dermatol 46 (2 Suppl Understanding): S63-S97, 2002.

2. Shockman S, Paghdal KV and Cohen G: Medical and surgical management of keloids: A review. J Drugs Dermatol 9: 1249-1257, 2010.

3. Wolfram D, Tzankov A, Pülzl P and Piza-Katzer H: Hypertrophic scars and keloids-a review of their pathophysiology, risk factors and therapeutic management. Dermatol Surg 35: 171-181, 2009.

4. Koike S, Akaishi S, Nagashima Y, Dohi T, Hyakusoku H and Ogawa R: Nd:YAG Laser treatment for keloids and hypertrophic scars: An analysis of 102 cases. Plast Reconstr Surg Glob Open 2: e272, 2014

5. Kumar K, Kapoor BS, Rai P and Shukla HS: In-situ irradiation of keloid scars with Nd:YAG laser. J Wound Care 9: 213-215, 2000.

6. Jones CD, Guiot L, Samy M, Gorman M and Tehrani H: The use of chemotherapeutics for the treatment of keloid scars. Dermatol Reports 7: 5880, 2015.

7. Trisliana Perdanasari A, Lazzeri D, Su W, Xi W, Zheng Z, Ke L, Min P, Feng S, Zhang YX and Persichetti P: Recent developments in the use of intralesional injections keloid treatment. Arch Plast Surg 41: 620-629, 2014 
8. van Leeuwen MC, Stokmans SC, Bulstra AE, Meijer OW, Heymans MW, Ket JC, Ritt MJ, van Leeuwen PA and Niessen FB: Surgical excision with adjuvant irradiation for treatment of keloid Scars: A systematic review. Plast Reconstr Surg Glob Open 3: e440, 2015.

9. Liu X, Wang H, Cen Y and Li Z: Synthetic therapy for keloid in aural region. Zhongguo Xiu Fu Chong Jian Wai Ke Za Zhi 22 56-58, 2008 (In Chinese).

10. Apikian M and Goodman G: Intralesional 5-fluorouracil in the treatment of keloid scars. Australas J Dermatol 45: 140-143, 2004

11. Bijlard E, Steltenpool S and Niessen FB: Intralesional 5-fluorouracil in keloid treatment: A systematic review. Acta Derm Venereol 95: 778-782, 2015.

12. Huang L, Cai YJ, Lung I, Leung BC and Burd A: A study of the combination of triamcinolone and 5-fluorouracil in modulating keloid fibroblasts in vitro. J Plast Reconstr Aesthet Surg 66: e251-e259, 2013.

13. Marneros AG and Krieg T: Keloids-clinical diagnosis, pathogenesis and treatment options. J Dtsch Dermatol Ges 2: 905-913, 2004.

14. Nanda S and Reddy BS: Intralesional 5-fluorouracil as a treatment modality of keloids. Dermatol Surg 30: 54-57, 2004.

15. Li J, Fu R, Li L, Yang G, Ding S, Zhong Z and Zhou S: Co-delivery of dexamethasone and green tea polyphenols using electrospun ultrafine fibers for effective treatment of keloid. Pharm Res 31: 1632-1643, 2014.

16. Kiil J: Keloids treated with topical injections of triamcinolone acetonide (kenalog). Immediate and long-term results. Scand J Plast Reconstr Surg 11: 169-172, 1977.

17. Callender VD, Young CM, Haverstock CL, Carroll CL and Feldman SR: An open label study of clobetasol propionate $0.05 \%$ and betamethasone valerate $0.12 \%$ foams in the treatment of mild to moderate acne keloidalis. Cutis 75: 317-321, 2005.

18. Yuan SM, Zhang M, Guo Y, Cui L, Hong ZJ and Jiang HQ: Intralesional injection of diprospan is effective for infantile hemangioma. J Craniofac Surg 26: 422-424, 2015

19. Muneuchi G, Suzuki S, Onodera M, Ito O, Hata Y and Igawa HH: Long-term outcome of intralesional injection of triamcinolone acetonide for the treatment of keloid scars in Asian patients. Scand J Plast Reconstr Surg Hand Surg 40: 111-116, 2006.

20. Roques $C$ and Téot L: The use of corticosteroids to treat keloids: A review. Int J Low Extrem Wounds 7: 137-145, 2008.
21. Wang XQ, Liu YK, Qing C and Lu SL: A review of the effectiveness of antimitotic drug injections for hypertrophic scars and keloids. Ann Plast Surg 63: 688-692, 2009.

22. Wang XQ, Liu YK, Wang ZY, Wei Jun, Jiang YZ, Qing Chun and Lu SL: Antimitotic drug injections and radiotherapy: A review of the effectiveness of treatment for hypertrophic scars and keloids. Int J Low Extrem Wounds 7: 151-159, 2008.

23. Darougheh A, Asilian A and Shariati F: Intralesional triamcinolone alone or in combination with 5-fluorouracil for the treatment of keloid and hypertrophic scars. Clin Exp Dermatol 34: 219-223, 2009.

24. Fitzpatrick RE: Treatment of inflamed hypertrophic scars using intralesional 5-FU. Dermatol Surg 25: 224-232, 1999.

25. Kontochristopoulos G, Stefanaki C, Panagiotopoulos A, Stefanaki K, Argyrakos T, Petridis A and Katsambas A: Intralesional 5-fluorouracil in the treatment of keloids: An open clinical and histopathologic study. J Am Acad Dermatol 52: 474-479, 2005

26. Abergel RP, Dwyer RM, Meeker CA, Lask G, Kelly AP and Uitto J: Laser treatment of keloids: A clinical trial and an in vitro study with Nd:YAG laser. Lasers Surg Med 4: 291-295, 1984.

27. Sherman $\mathrm{R}$ and Rosenfeld H: Experience with the Nd:YAG laser in the treatment of keloid scars. Ann Plast Surg 21: 231-235, 1988.

28. Alster TS and Williams CM: Treatment of keloid sternotomy scars with $585 \mathrm{~nm}$ flashlamp-pumped pulsed-dye laser. Lancet 345: 1198-1200, 1995.

29. Connell PG and Harland CC: Treatment of keloid scars with pulsed dye laser and intralesional steroid. J Cutan Laser Ther 2: 147-150, 2000.

30. Lawrence WT: In search of the optimal treatment of keloids: Report of a series and a review of the literature. Ann Plast Surg 27: 164-178, 1991

31. Manuskiatti W and Fitzpatrick RE: Treatment response of keloidal and hypertrophic sternotomy scars: Comparison among intralesional corticosteroid, 5-fluorouracil and 585-nm flashlamp-pumped pulsed-dye laser treatments. Arch Dermatol 138: 1149-1155, 2002. 